\title{
Berberine Inhibits Pro-inflammatory Cytokine-induced IL-6 and CCL11 Production via Modulation of STAT6 Pathway in Human Bronchial Epithelial Cells
}

\author{
Jason $\mathrm{Ma}^{1 *}$, Cheng-Chi Chan ${ }^{2 *}$, Wen-Chung Huang ${ }^{3,4,5}$ and Ming-Ling Kuo ${ }^{1,4,5}$ \\ 1. Department of Microbiology and Immunology, Graduate Institute of Biomedical Sciences, College of Medicine, Chang Gung University, Taoyuan, Taiwan \\ 2. Cancer Vaccine and Immune Cell Therapy Core Laboratory, Chang Gung Immunology Consortium, Chang Gung Memorial Hospital, Linkou, Taoyuan, \\ Taiwan. \\ 3. Graduate Institute of Health Industry Technology, Research Center for Food and Cosmetic Safety, College of Human Ecology, Chang Gung University of \\ Science and Technology, Taoyuan City, Taiwan. \\ 4. Research Center for Chinese Herbal Medicine, College of Human Ecology, Chang Gung University of Science and Technology, Taoyuan City, Taiwan. \\ 5. Division of Allergy, Asthma, and Rheumatology, Department of Pediatrics, Chang Gung Memorial Hospital, Taoyuan, Taiwan. \\ *These authors contributed equally to this work.
}

$\square$ Corresponding author: Dr. Ming-Ling Kuo, Department of Microbiology and Immunology, College of Medicine, Chang Gung University, 259 Wen-Hwa 1st Road, Kwei-Shan, Tao-Yuan, TAIWAN. TEL: 886-3-2118800, ext 3319, FAX: 886-3-2118293, E-mail: mingling@mail.cgu.edu.tw.

(c) The author(s). This is an open access article distributed under the terms of the Creative Commons Attribution License (https://creativecommons.org/licenses/by/4.0/). See http://ivyspring.com/terms for full terms and conditions.

Received: 2020.02.27; Accepted: 2020.05.29; Published: 2020.06.08

\begin{abstract}
Berberine is an isoquinoline alkaloid isolated from various Chinese herbs that has potential of antiinflammatory, anti-lipidemic, anti-neoplastic, and anti-diabetic activity. In this study, we evaluated the anti-inflammatory efficacy of berberine on allergic airway inflammation by targeting epithelial cells. Allergic airway inflammation driven by $\mathrm{T}$ helper 2 (Th2)-type immunity is characterized by airway hyperresponsiveness, elevated IgE production, and eosinophilic infiltration. For eosinophil recruitment, major chemoattractant CCL11 (eotaxin-1) was secreted by lung epithelial cells. BEAS-2B cells, a human bronchial epithelial cell line, were pre-treated with berberine and then activated by IL-4 plus TNF- $\alpha$. The viability of BEAS-2B cells was assessed. Expression levels of IL-6 and CCLI 1 were determined using ELISA and real-time PCR. The signaling pathways of MAP kinases, NF-KB, and STAT6 were analyzed by western blot. Berberine treatment $(\leq 1 \mu \mathrm{M})$ didn't significantly affect the viability of BEAS-2B cells with or without IL-4 plus TNF-stimulation. Berberine significantly inhibited the secretion of IL-6 and CCL11 from pro-inflammatory cytokine-activated BEAS-2B cells. NF-KB and MAP kinase pathways were seemingly unaffected in BEAS-2B cells with berberine treatment. Significant reduction of nuclear STAT6 protein expression in activated BEAS-2B cells with berberine treatment was observed. Current study reveals that berberine has inhibitory effect in pro-inflammatory cytokine-activated BEAS-2B cells through reducing IL-6 and CCL11 production, which is possibly modulated by suppressing STAT6 signaling pathway.
\end{abstract}

Key words: Berberine, cytokines, asthma, eotaxin, epithelial cell

\section{Introduction}

Asthma is one of the major public health concerns worldwide, with high prevalence and economic burden [1]. Asthma is a chronic inflammatory airway disease characterized by airflow obstruction, persistent inflammation, and airway hyperresponsiveness (AHR) to common environmental aero-allergens [2]. Asthmatic patients exhibit elevated allergen-specific IgE, mucus hypersecretion, and eosinophilia in the lungs. Most treatments for asthma are based on western medicine, such as $\beta 2$-adrenergic receptor agonists, anti- $\operatorname{IgE}$ blockers, and corticosteroids combined with a longacting bronchodilator, but only provide temporary alleviation of the symptoms [3]. In addition, steroids have certain side effects and some patients do not favorably respond to this treatment. Nevertheless, traditional Chinese medicines are reported to have 
improved or curative effects in clinical trials for allergic diseases including asthma [4].

Airway epithelium acts as the first defense barrier against environmental stimuli by secreting numerous chemokines, cytokines and growth factors to recruit and activate a variety of immune cells including $\mathrm{CD}^{+} \mathrm{T}$ helper 2 (Th2) cells, mast cells, and eosinophils [5]. Th2 cytokines, especially interleukin (IL)-4, IL-5, and IL-13, and other inflammatory mediators secreted by eosinophils and mast cells are primarily thought to orchestrate pathological features of asthma [6]. CCL11 (eotaxin-1), the most potent chemokine for eosinophil movement, is secreted by bronchial epithelial and endothelial cells and its receptor, CCR3, is highly expressed on eosinophils [7]. CCL11 is also verified to activate eosinophils by eliciting the release of RNase and cell-free granules [8]. Significant increase of CCL11 levels is detected in blood, sputum, and exhaled breath condensate (EBC) of asthmatic patients compared with healthy subjects [9]. Moreover, the disruption of CCL11 gene expression effectively reduces tissue eosinophilia [10]. Therefore, the reduction of eosinophil recruitment by inhibiting CCL11 expression in the lungs might have therapeutic potential for asthma.

The level of pro-inflammatory cytokine IL-6 was elevated in asthma patients [11]. A large variety of cells can produce IL-6, including cells from innate immune system, endothelial cells, fibroblasts, epithelial cells, etc. [12]. IL-6 functions as a growth factor for B cells and also contributes to CD4 T cells differentiation [13]. The presence of IL-6 promotes autocrine IL-4 production by activating STAT3 and ERK pathway [14], IL-5 production by p38 pathway, with the help of IL-2 signaling [15], and further enhances Th2 differentiation through an autofeedback loop. Nonetheless, elevation of IL-6 is also correlated with elevated level of IL-13 and increased in asthma patients [16]. Taken together, targeting IL-6 may alleviate asthmatic responses by obstructing Th2 differentiation.

Previous papers showed that IL-4 and tumor necrosis factor (TNF)- $\alpha$ can stimulate the fibroblasts of nasal polyps to produce eotaxin-1 [17, 18]. Similarly, other evidences reveal that IL-4 plus TNF-a triggers the eotaxin secretion of human bronchial epithelial cells, BEAS-2B [19]. The activation of eotaxin gene expression is regulated by NF-KB and STAT6 in human airway epithelial cells upon IL-4 activation [19]. NF-kB pathway is also involved in regulating diverse inflammatory cytokine genes including IL-1 $\beta$ and TNF- $\alpha$ that re-stimulate immune cells and epithelial cells to exacerbate the inflammation [20]. With TNF- $\alpha$, MAP kinases, which comprise the extracellular signal-regulated kinases (ERK), the p38
MAP kinases, and the c-Jun NH2-terminal kinases (JNK), can also be activated [21]. MAP kinase signaling pathways transduce a variety of extracellular signals that mediate cellular functions implicated in proliferation, differentiation, inflammation, and apoptosis [22].

Previous studies have reported that berberine, an alkaloid extracted from various traditional Chinese herbs, has pharmacological effects on intestinal bacteria, parasites, cardiovascular and cerebrovascular diseases, lowering blood sugar, and cancer [23]. In respiratory tract infections, berberine has been confirmed to suppress inflammatory agents, such as IL-1 $\beta$ and TNF- $\alpha$ by inhibiting IKB degradation in human lung cells [24]. Several papers also indicate that berberine decreases the inflammatory response by repressing the inflammation cytokine expression in colonic macrophages and epithelial cells or retinal pigment epithelial cells by down-regulating transcription factor NF-kB or the MAP kinase pathways [25, 26]. Based on these evidences, berberine is seemly capable of suppressing the inflammatory responses.

In present study, we applied BEAS-2B cells, a human bronchial epithelial cell line, as an in vitro model to examine the anti-inflammatory efficacy of berberine on pro-inflammatory cytokine-stimulated epithelial cells. NF-kB, STAT6 and MAP kinases signaling pathways involved in modulating eotaxin gene expression are assessed.

\section{Materials and Methods}

\section{Materials}

Figure 1A shows the chemical structure of berberine chloride ( $\geq 98 \%$ purity by TLC; SigmaAldrich). A stock solution of $20 \mathrm{mM}$ berberine was prepared in DMSO (Sigma-Aldrich). The final DMSO concentration did not exceed $0.1 \%$ in the culture medium.

\section{Cell culture and berberine treatment}

Human bronchial epithelial cells (BEAS-2B CRL9609 cell line, ATCC) were cultured in Dulbecco's Modified Eagle Medium/Nutrient mixture F-12 (DMEM/F12) medium (Gibco) containing 10\% fetal bovine serum (FBS, Gibco) and 1\% penicillin/ streptomycin (Gibco). Cells were seeded in 6-well plates $\left(2 \times 10^{6}\right.$ cells) or 24 -well plates $\left(4 \times 10^{5}\right.$ cells) at 37 ${ }^{\circ} \mathrm{C}$ in a humidified $5 \% \mathrm{CO}_{2}$ atmosphere. Berberine chloride (Sigma-Aldrich) was dissolved in DMSO (Sigma-Aldrich), where same amount of DMSO for dissolving berberine was used as control for comparison. BEAS-2B cells were treated with or without different concentrations of berberine for 16-18 hours. After pre-treatment, BEAS-2B cells were 
stimulated with IL-4 (20 ng/ml) (Peprotech) plus TNF-a $(5 \mathrm{ng} / \mathrm{ml})$ (Peprotech) and culture supernatants were harvested after 6 hours and 24 hours to detect IL-6 and CCL11 levels by ELISA assay. For inhibition assays, $10 \mu \mathrm{M}$ of JNK inhibitorSP600125 (Enzo) and ERK inhibitor-PD-98059 (Enzo) were added one hour before cytokine stimulation and culture supernatants were harvested after 24 hours for IL-6 and CCL11 detection.

\section{Cell viability assay}

BEAS-2B cells $\left(2 \times 10^{5}\right.$ cells $)$ were seeded in a 48-well plate overnight and then were pre-treated with various concentrations of berberine or DMSO for 16-18 hours. After pre-treatment, cells were stimulated without or with IL-4 and TNF-a for 24 hours and the viability was analyzed by Cell Counting-kit 8 (CCK-8) (Sigma-Aldrich). Briefly, after the supernatants were harvested, cells were washed with $1 \mathrm{ml}$ PBS and then CCK-8 was added into each well to incubate at $37{ }^{\circ} \mathrm{C}$ in a humidified $5 \% \mathrm{CO}_{2}$ atmosphere for 4 hours. Supernatants were transferred into a 96-well plate after incubation. Absorbance was measured on an ELISA reader at 450 nm (SpectraMax).

\section{Enzyme-linked immunosorbent assay}

Culture supernatants were harvested following 6 or 24 hours of IL-4 and TNF-a stimulation. The levels of IL-6 and CCL11 (DuoSet ELISA kit, R\&D systems) were determined by ELISA according to the manufacturer's instructions.

\section{RNA isolation and real-time polymerase chain reaction}

RNA samples were collected at 3, 6, 12 and 24 hours after cytokine stimulation. Total RNA was extracted using TRIzol reagent (Invitrogen). Reverse transcription was performed by oligo-dT primers and M-MLV reverse transcriptase (Invitrogen). The mRNA expression of GAPDH, IL-6, and CCL11 were amplified by specific primers: 5'-GCAAATTCCATGG CACCG-3' for GAPDH forward primer and 5'-TCGC CCCACTGATTTTGG-3' for GAPDH reverse primer; 5'-CCAATCTGGATTCAATGAGGAG-3' for IL-6 forward primer and 5'-GGTCAGGGGTGGTTATTGC ATC-3' for IL-6 reverse primer; 5'-AAAGCTCACACC TTCAGCCT-3' for CCL11 forward primer and 5'-TTT CTGGGGACATTTGCCAC-3' for CCL11 reverse primer. For real-time PCR, the reaction was performed by Lightcycler ${ }^{\circledR} 96$ system (Roche) with $\mathrm{iQ}^{\mathrm{TM}}$ SYBR green supermix (Bio-rad). The relative expression of each gene was calculated by normalizing to the expression of GAPDH.

(a)<smiles>COc1ccc2cc3[n+](cc2c1OC)CCc1cc2c(cc1-3)OCO2</smiles>

(b)

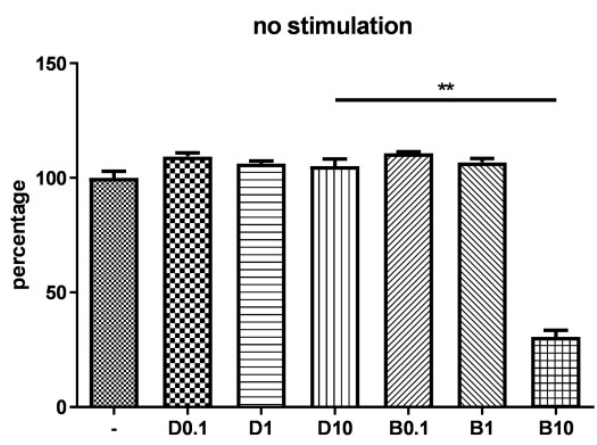

(c)

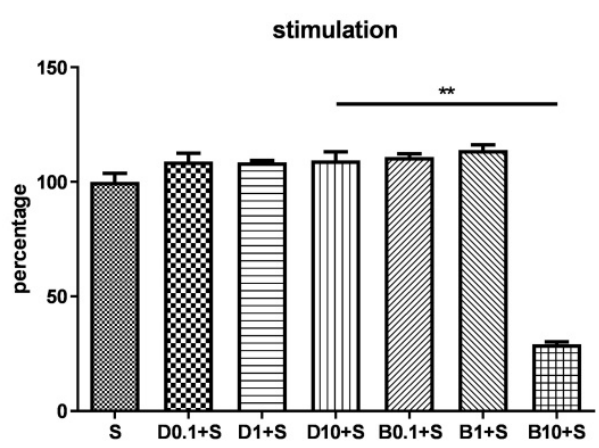

Figure 1. Cytotoxicity of berberine on human bronchial epithelial cell line. (A) The chemical structure of berberine. BEAS-2B cells were cultured in the 48-well plates overnight and then were treated with different concentrations of berberine $(0.1 \mu \mathrm{M}$ to $10 \mu \mathrm{M})$ or equal volume of DMSO for 16 to 18 hours. Subsequently, drug pre-treated cells were stimulated (A) without or (B) with pro-inflammation cytokines for 24 hours. The cell viability was analyzed by CCK-8 assay. The percentage was calculated by comparing the O.D. value with cell only group. Data are presented as mean \pm SEM $(n=6)$. S, pro-inflammation cytokine stimulation; D, DMSO; B, berberine. The number indicated the concentration $(\mu \mathrm{M})$ of berberine or DMSO. $* * P<0.01$. 


\section{The extraction of total cell, cytoplasm and nucleus protein}

For total cell protein extraction, protein samples were collected at indicated time point after cytokine stimulation. Cells were lysed in cell lysis buffer containing $1 \mathrm{mM} \mathrm{Na} \mathrm{VO}_{4}, 1 \mathrm{mM}$ Tetranisole, 25X diluted protease inhibitor, $5 \mu \mathrm{M} \mathrm{MgCl}_{2}, 20 \mu \mathrm{M}$ Tris- $\mathrm{HCl}, 50 \mu \mathrm{M} \mathrm{NaCl}, 20 \% \mathrm{NP}-40$ on ice for $30 \mathrm{~min}$. Cell lysates were centrifuged at $12,000 \mathrm{~g}$ for $30 \mathrm{~min}$ at $4{ }^{\circ} \mathrm{C}$ and the supernatants were harvested for western blot analysis. For cytoplasm and nucleus protein extraction, cell extracts were collected using Nuclear and Cytoplasmic Extraction Reagent kit (NE-PER kit) (Pierce Biotechnology) according to the manufacturer's instructions. Protein concentrations were determined using a BCA protein assay (Pierce Biotechnology) for western blot analysis.

\section{Western blot analysis}

Protein samples $(20 \mu \mathrm{g})$ were separated on sodium dodecyl sulfate-polyacrylamide gel, transferred to nitrocellulose membrane (Millipore) at $50 \mathrm{~mA}$ for $30 \mathrm{~min}$ and blocked in Tris-buffered saline with $0.1 \%$ Tween 20 (TBST) containing 5\% BSA at room temperature for 1 hour. The blots were incubated with primary antibodies (rabbit anti-human antibody) to JNK, phosphorylated JNK, ERK, phosphorylated ERK, p38, phosphorylated p38, IкB, phosphorylated IкB, p65, STAT6, and phosphorylated STAT6, primary antibodies (mouse anti-human antibody) for $\beta$-actin and p38, and primary antibodies (goat anti-human antibody) for PCNA (Cell Signaling) overnight at $4{ }^{\circ} \mathrm{C}$. Secondary antibodies conjugated with horseradish peroxidase were incubated for 2 hours. Finally, the blots were developed by using the Immobilon Western chemiluminescent HRP substrate (Millipore).

\section{Statistical analysis}

Results are presented as mean \pm SEM. Significance was assessed using a two-tailed $P$-value calculated by the Mann-Whitney $U$ test. $P<0.05$ is considered significant. All graphs were generated and statistical analyses performed using GraphPad Prism 8.0 software.

\section{Results}

\section{Determine berberine concentration for BEAS-2B without significant cytotoxic effect}

Before examining anti-inflammatory efficacy of berberine, we evaluated whether berberine has cytotoxicity on BEAS-2B cell line. Cells were pre-treated with different concentrations of berberine $(0.1 \mu \mathrm{M}-10 \mu \mathrm{M})$ or DMSO as control. Cells were stimulated with or without IL-4 plus TNF-a for activation and cell viability was assayed. The data indicated berberine at $1 \mu \mathrm{M}$ or lower concentrations didn't affect the viability of BEAS-2B cells with or without pro-inflammatory cytokine stimulation compared with DMSO control (Figure 1B and C). Berberine had certain cytotoxic effect on BEAS-2B cells when treated with berberine higher than $10 \mu \mathrm{M}$ (Figure 1 and data not shown). Corresponded concentrations of DMSO control to berberine had no toxicity on BEAS-2B cells. Thus, in subsequent experiments, we used $1 \mu \mathrm{M}$ berberine to evaluate the anti-inflammatory effect of berberine on BEAS-2B cells.

\section{Berberine significantly reduced the production of IL-6 and CCLI 1 in pro-inflammatory cytokine-activated BEAS-2B cells}

To assess the role of berberine on suppressing inflammatory mediators in bronchial epithelial cells, BEAS-2B cells were stimulated with IL-4 plus TNF-a for 6 and 24 hours. BEAS-2B cells without pro-inflammatory cytokine stimulation secreted low levels of IL-6 (Figure 2A and B) and CCL11 (Figure 2C and D). Significant amounts of IL-6 and CCL11 were detected in BEAS-2B cells after IL-4 plus TNF-a stimulation. Berberine $(1 \mu \mathrm{M})$ significantly suppressed the protein levels of IL-6 (Figure 2A and B) and CCL11 (Figure 2C and D) in stimulated BEAS-2B cells compared to untreated group.

To carefully examine the gene expression, total RNA were collected from stimulated BEAS-2B cells with berberine pre-treatment at different time points and the gene expression of IL-6 and CCL11 were analyzed by real-time PCR (Figure 3). Obvious RNA expression of IL-6 and CCL11 were detected in BEAS2B cells with pro-inflammatory cytokine stimulation (Figure $3 \mathrm{~A}$ and $\mathrm{B}$ ). Berberine treatment reduced the expression levels of IL-6 and CCL11 genes in stimulated BEAS-2B cells, which is consistent with cytokine secretion pattern. These results indicated that reduced inflammatory mediators (IL-6 and CCL11) in response to berberine pre-treatment may alleviate activated BEAS-2B cells-mediated airway inflammation.

\section{Significant reduction of nuclear STAT6 protein expression in activated BEAS-2B cells with berberine treatment}

To investigate possible mechanisms involved in berberine-suppressed cytokine production, we first dissected the expression of STAT6 in BEAS-2B cells. Previous studies demonstrated that eotaxin-1 (CCL11) can be secreted from airway epithelial cells and fibroblasts after IL-4 plus TNF-a stimulation [17]. The 
activation of STAT6 pathways play a pivotal role in regulating the expression of CCL11 gene [19]. We analyzed cytoplasmic and nuclear STAT6 in activated cells with or without berberine treatment by western blotting (Figure 4A). In both cytoplasm (Figure 4B) and nucleus (Figure 4C), lower STAT6 protein expresion was observed after berberine treatment in activated BEAS-2B cells, while significantly decreased STAT6 protein was detected 6 hours after stimulation in the nucleus. Phosphorylated STAT6 expression was also decreased in nucleus after 60 minutes (Figure S1). These results suggest that berberine may reduce the expression of nuclear STAT6 protein in activated BEAS-2B cells to suppress the CCL11 production.

\section{Effect of berberine on MAP kinase pathways in activated BEAS-2B cells}

To investigate how berberine suppressed IL-6 production in activated BEAS-2B cells, the expression and activity of MAP kinases and NF-kB were examined. The expression of p-JNK1/JNK1, p-JNK2/ JNK2, p-ERK1/ERK1, and p-ERK2/ERK2 were detected in BEAS-2B cells with IL-4 plus TNF-a stimulation (Figure 5A and 6A). The expression levels of JNK and ERK proteins were not different between each group after stimulation (Figure $5 \mathrm{~A}$ and $6 \mathrm{~A}$ ). Relative expression levels of $\mathrm{p}$-JNK1/JNK1 remains no change after berberine treatment, while p-JNK2/ JNK2 (Figure 5B) was elevated 15 minutes after stimulation but later was recovered (Figure 5C). On the other hand, p-ERK1/ERK1, and p-ERK2/ERK2 (Figure 6B and C) were elevated in activated BEAS-2B cells with berberine treatment compared to untreated groups, but did not show significant difference. No significant differences were detected in p38 protein expression in activated BEAS-2B cells with or without berberine treatment and p-p38 expression levels were undetectable among the different groups (data not shown). We confirmed this result by adding JNK inhibitor or ERK inhibitor into berberine-treated and cytokine-activated BEAS-2B cells. The level of IL-6 (Figure S2A) and CCL11 (Figure S2B) were still suppressed in berberine treated group after using JNK and ERK inhibitors. Thus, berberine did not obviously affect the MAP kinase pathways of activated BEAS-2B cells. Also, berberine did not influence the expression levels of IкB (Figure 7B), p-IкB (Figure 7C) or p65 (Figure 7D), a subunit of NF- $\mathrm{kB}$, in activated BEAS-2B cells.
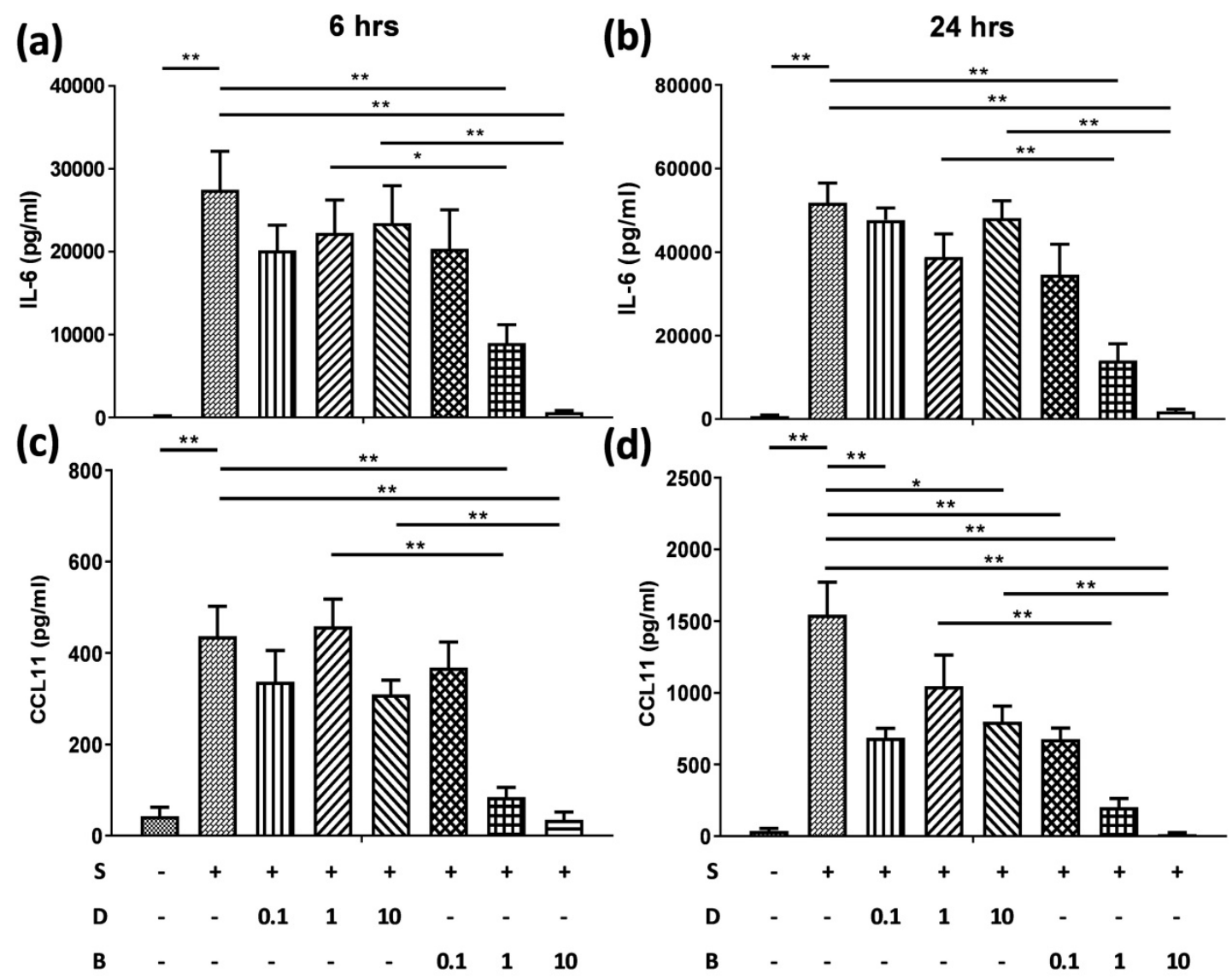

Figure 2. Berberine pre-treatment suppressed pro-inflammatory cytokine-induced IL-6 and CCLII production in the BEAS-2B cells. BEAS-2B cells were seeded in the 48 -well plate and treated with berberine $(0.1 \mu \mathrm{M}$ to $10 \mu \mathrm{M})$ or DMSO (control group) overnight. Cells with berberine pre-treatment were activated without or with IL-4 plus TNF- $\alpha$ for 6 and 24 hours. Culture supernatants were harvested and measured for (A, B) IL-6 and (C, D) CCL11 using ELISA. Data are presented as mean \pm SEM $(n=6)$. S, pro-inflammation cytokine stimulation; D, DMSO; B, berberine. The number indicated the concentration $(\mu M)$ of berberine or $D M S O$. $* P<0.05$; $* * P<0.01$. 
(a)
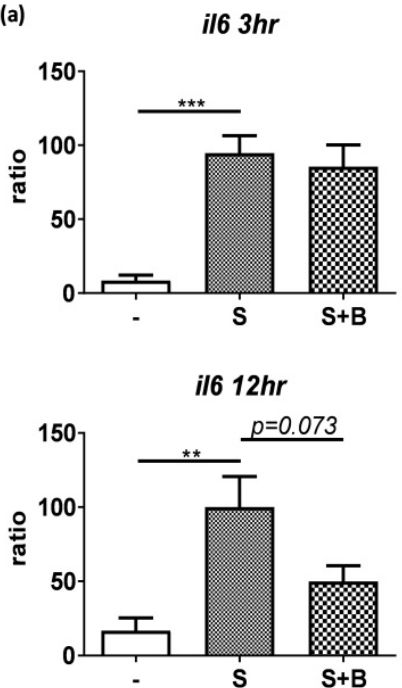

(b)
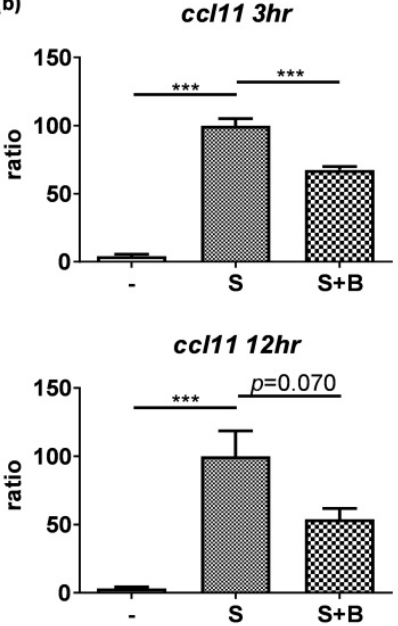
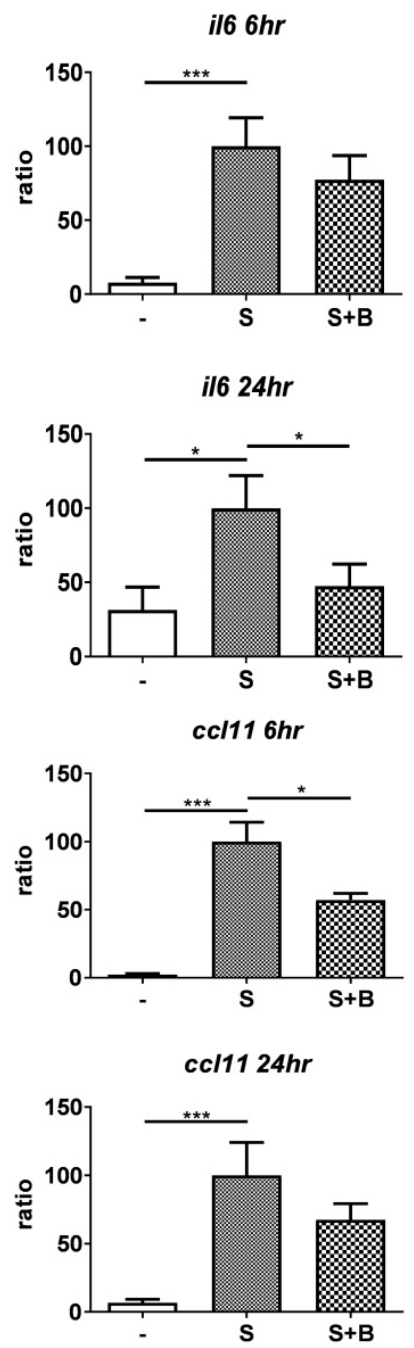

Figure 3. Expression levels of IL-6 and CCLII gene were reduced in pro-inflammatory cytokine-stimulated BEAS-2B cells with berberine pre-treatment. Total RNA was extracted from IL-4 plus TNF- $\alpha$-activated BEAS-2B cells pre-treated with berberine $(1 \mu \mathrm{M})$ at $3,6,12$, and 24 hours. The RNA expression levels of (A) IL-6 and (B) CCL1 1 detected by real-time PCR, normalized with GAPDH and compared with stimulation group $(n=7)$. Data are presented as mean \pm SEM. -, cell only; S, pro-inflammation cytokine stimulation; B, berberine. $* P<0.05, * * P<0.01, * * * P<0.001$

\section{Discussion}

Allergic asthma is a chronic inflammatory disease of the airways that has become a global public health issue to result in a considerable burden on health services and expensive costs. The bronchial epithelium is recognized as a regulator of the initiation and maintenance of allergic airway inflammation [5]. Despite a growing number of pharmaceutical and immunotherapy strategies for asthma, the development of effective and long-term immunomodulatory approach has been difficult. In many Asian countries, traditional Chinese medicine is routinely used as maintenance of daily health care or as complementary treatment for conventional

Western medicine. Many evidences showed that traditional Chinese herb medicine has improved or therapeutic efficacy in allergic diseases, such as asthma, atopic dermatitis, and food allergy [27-29]. Here, our results demonstrated that berberine is capable of suppressing the secretion of IL-6 and CCL11 in pro-inflammatory cytokine-activated BEAS-2B cells, human airway epithelial cells. The inhibition of pro-inflammatory cytokine-induced IL-6 and CCL11 production by berberine may cause by reduction of nuclear STAT6 expression in activated cells. Consequently, berberine may be applied as a candidate for the improvement or treatment of allergic asthma.

Corticosteroids and bronchodilators are the most common treatment of asthma [30]. However, a few asthmatic patients respond poorly to these drugs or require higher doses to control the symptoms that lead to certain side-effects [31]. Some patients seek to improve asthma symptoms using alternative medicine, including Chinese herbal medicine, yoga, homeopathy, and even urine therapy [32]. Ma Xing Gan Shi Tang, Xiao Qing Long Tang, and Ding Chuan Tang are popular asthmatic complementary and alternative treatments which can modulate Th2 celldriven airway inflammation [29, 33, 34]. However, these herbal formulas contain Ephedra spp., which contains ephedrine, causes excitement and unwanted side effects [35]. Hence, other Chinese herbal formulas, single components, or pure compound extracts have been proved to relieve or treat the asthmatic symptoms, such as phloretin [36], ASHMI [37], MSSM-002 [38], and CVT-E002 [39]. ASHMI, which is composed by Sophora flavescens, Glycyrrhiza uralensis, and Ganoderma lucidum, suppresses AHR, airway eosinophilia, and Th2 cell-secreted cytokines in asthmatic mice [37]. ASHMI was also successfully applied for the Phase I of clinical trial to ameliorate FEV1, peak expiratory flow, and serum IgE levels [40]. Extract from plants or compounds also have therapeutic effects on asthma. For example, extracts of Nigella sativa or purple passion fruit peel were also reported to reduce asthmatic symptoms like cough, wheeze, and shortness of breath in asthmatic patients $[41,42]$. Baicalin, a flavonoid compound isolated from Scutellaria baicalensis, was reported to suppress STAT3 expression and promotes FoxP3 expression to alleviate asthmatic symptoms in mice [43]. Therefore, single components or pure compound extracts from various Chinese herbs are able to serve as an attractive approach to modulate allergic asthma. 
(a)
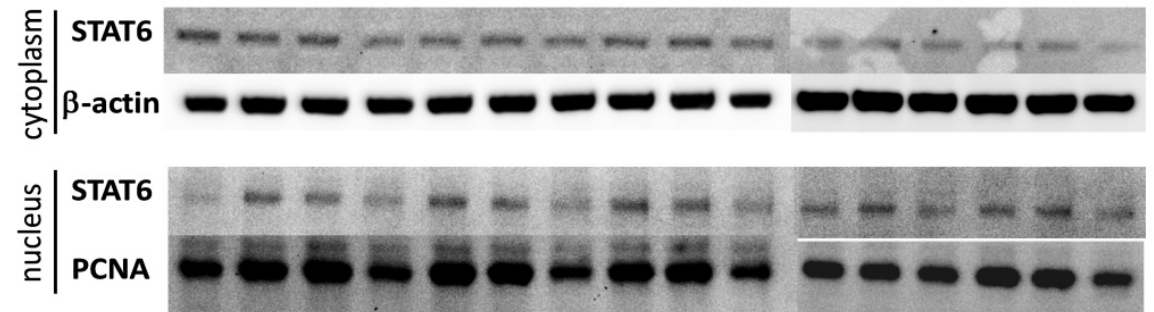

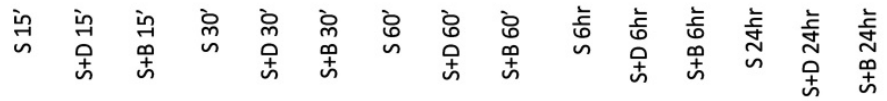

(b)

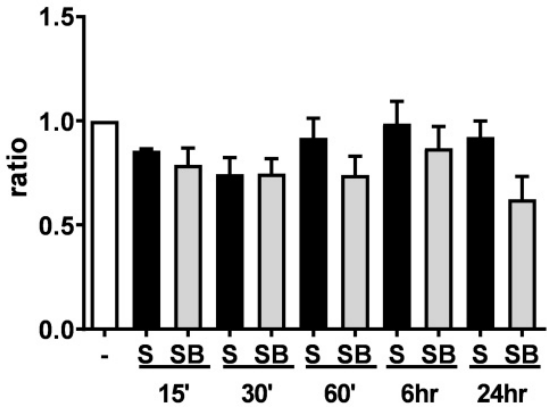

(c)

STAT6 (N)

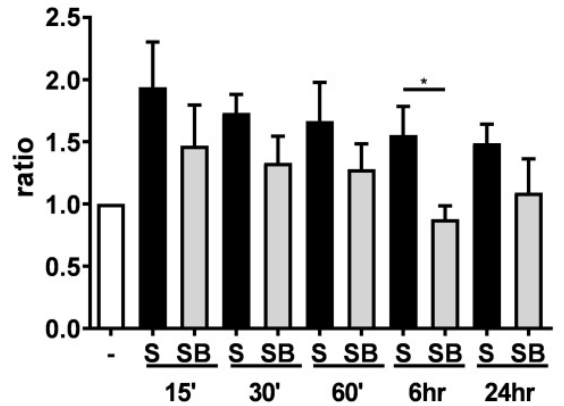

Figure 4. Berberine down-regulates STAT6 protein expression in nucleus with pro-inflammatory cytokine stimulated BEAS-2B cells. Cells with berberine (1 $\mu \mathrm{M}$ ) treatment were harvested on the indicated time points after IL-4 plus TNF- $\alpha$ stimulation and cytoplasm and nucleus proteins were extracted. (A) Expression levels of cytoplasm and nucleus STAT6 proteins were analyzed using western blotting (20 $\mathrm{gg}$ per sample). $\beta$-actin and PCNA expression was used as an internal control. The relative quantity of $(B)$ cytoplasm STAT6 $(n=4)$ and $(C)$ nucleus STAT6 $(n=7)$ was normalized to $\beta$-actin and PCNA, respectively. Results are presented as mean \pm SEM. *P<0.05. -, cell only; S, pro-inflammation cytokine stimulation; D, DMSO, B, berberine.

(a)

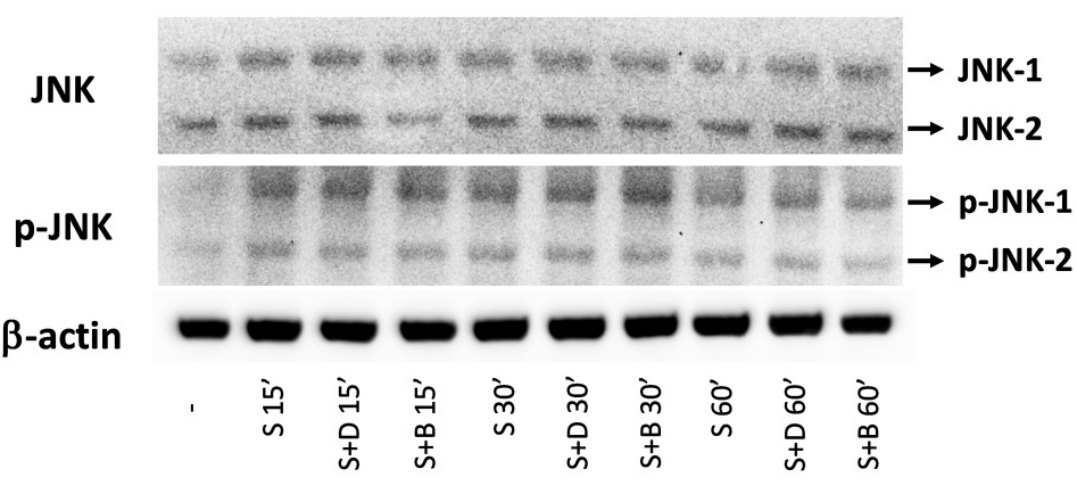

(b)

p-JNK1/JNK1

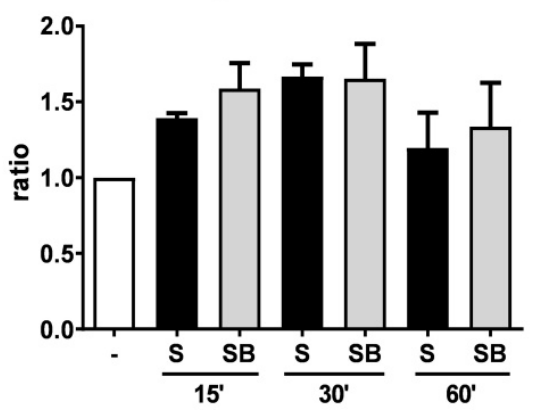

(c)

p-JNK2/JNK2

Figure 5. JNK and p-JNK protein expression of activated BEAS-2B cells pre-treated with or without berberine. Total cell proteins were collected at 15,30 , and 60 min of pro-inflammatory cytokine stimulation in BEAS-2B cells with berberine $(1 \mu \mathrm{M})$ pre-treatment. (A) Expression levels of JNK and p-JNK proteins were analyzed using western blotting $(20 \mu \mathrm{g}$ per sample). The relative quantity of (B) P-JNK1 and (C) P-JNK2 was normalized to JNK1 and JNK2, respectively. $\beta$-actin expression was used as an internal control. Results are presented as mean \pm SEM of six independent experiments. -, cell only; S, pro-inflammation cytokine stimulation; D, DMSO, B, berberine. 
(a)

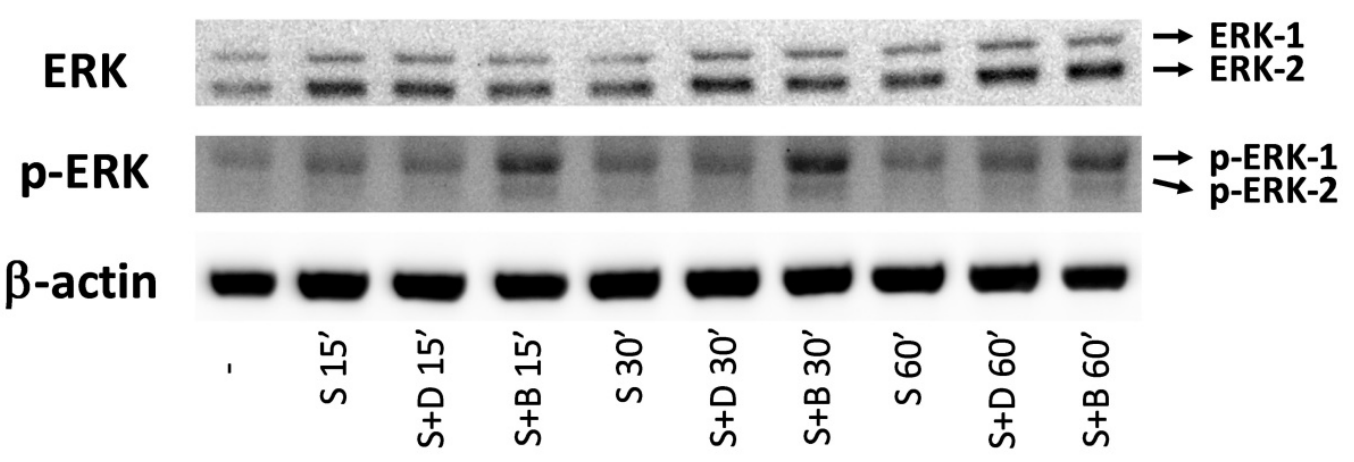

(b)

(c)

p-ERK1/ERK1

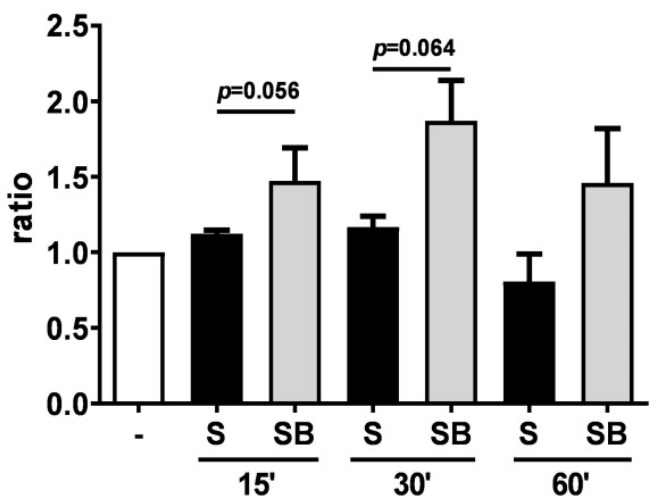

p-ERK2/ERK2

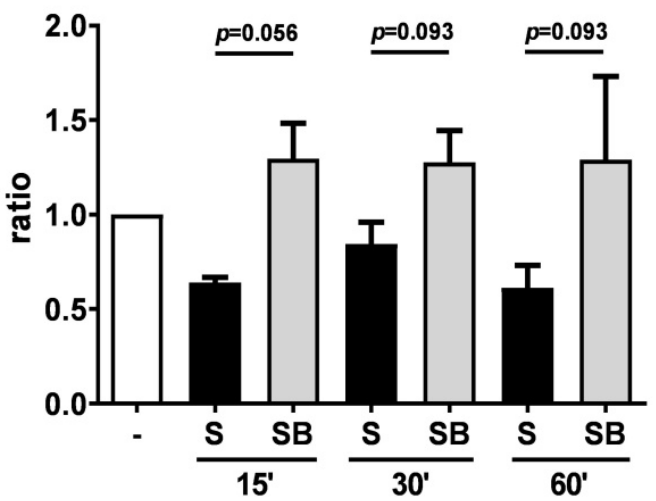

Figure 6. Western blot analysis of ERK and p-ERK in pro-inflammatory cytokine stimulated BEAS-2B cells with berberine pre-treatment. Total cell proteins were collected at 15, 30, and 60 min of IL-4 plus TNF- $\alpha$ stimulation in BEAS-2B cells with berberine $(1 \mu M)$ pre-treatment. (A) Expression levels of ERK and p-ERK proteins were analyzed using western blotting ( $20 \mu \mathrm{g}$ per sample). The relative quantity of (B) p-ERK1 and (C) p-ERK2 was normalized to ERK1 and ERK2, respectively. $\beta$-actin expression was used as an internal control. Results are presented as mean \pm SEM of six independent experiments. -, cell only; S, pro-inflammation cytokine stimulation; D, DMSO, B, berberine.

Alkaloid-containing plants have been used as medicine for animal and human starting from 4000 years ago. Alkaloids and derived species have been widely used to treat a variety of illnesses [44]. Berberine, an isoquinoline alkaloid that can be extracted from various Chinese herbs such as Coptis chinensis, Hydrastis canadensis, Berberis aristata and others, has potential of anti-inflammatory, anti-lipidemic, anti-neoplastic, and anti-diabetic activity [23, 45]. A previous study showed that berberine $(100 \mu \mathrm{M})$ was not toxic to A-549, U-937, and HFL-1 cell lines [24]. Our results indicate that berberine has dose-dependent cytotoxicity in BEAS-2B cells, although berberine at lower than $1 \mu \mathrm{M}$ is innocuous. At this concentration, berberine significantly reduced the secretion of IL- 6 and CCL11 in IL-4 plus TNF-a-activated BEAS-2B cells. The activation of eotaxin-1 gene expression in IL-4 plus TNF-a-stimulated airway epithelial cells and fibroblasts was regulated by activating JAK1/3-STAT6 pathway [19]. After phosphorylation, STAT6 form homodimers and enter the nucleus [46]. A report has demonstrated that berberine inhibits IL-2 induced JAK3 phosphorylation in monoarhritic rats [47]. In our results, berberine significantly repressed the expression of nuclear STAT6 in activated BEAS-2B cells and reduces CCL11 levels. In ovalbumin (OVA)-induced rat model of asthma, berberine has been reported to relieve inflammatory cell infiltration, lung inflammation, and $\operatorname{IgE}$ production [48]. The suppressive effects on the airway inflammation might be mediated through the inhibition of NF-KB signaling pathway by berberine treatment. Berberine blocks the caspase1/NF-kB pathway to reduce thymic stromal lymphopoietin (TSLP) production in human mast cell line, HMC-1 cells [49]. However, in activated BEAS-2B cells, berberine didn't decrease the

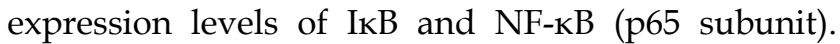
Berberine was also proposed to induce the production of IL-12 p40 by activating p38 MAP kinase in mouse macrophages [50]. In ARPE-19 cells, IL-6 secretion was stimulated by TNF-a through p38 MAP kinase, while berberine down-regulated the phosphorylation of p38 MAP kinase [26]. Although JNK and ERK might be elevated after berberine treatment, we demonstrated that the decrease of IL- 6 and CCL11 
(a)
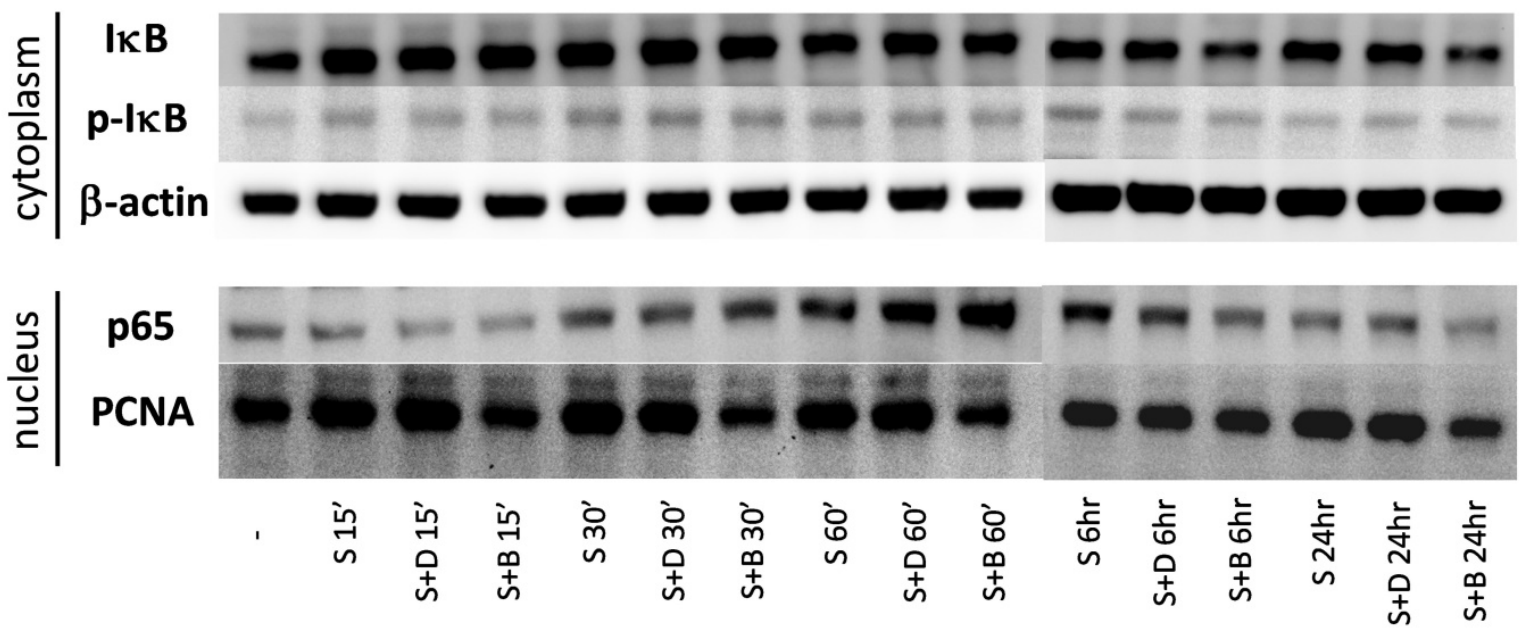

(b)

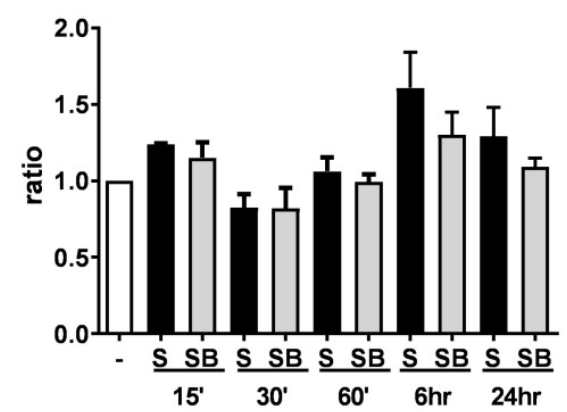

(c)

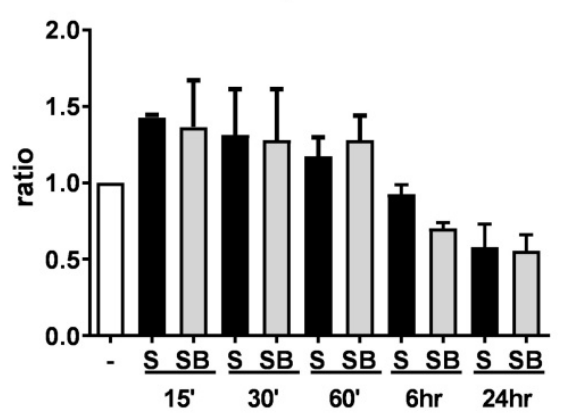

(d)

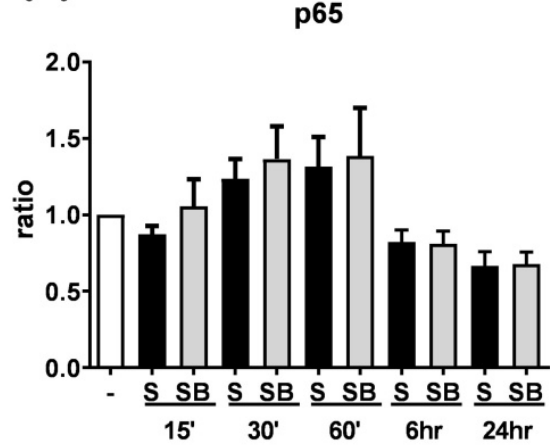

Figure 7. Western blot analysis of IKB, p-IKB, and p65 in pro-inflammatory cytokine stimulated BEAS-2B cells with berberine pre-treatment. Total cell proteins were collected at $15 \mathrm{~min}, 30 \mathrm{~min}, 60 \mathrm{~min}, 6$ hours, and 24 hours of IL- 4 plus TNF- $\alpha$ stimulation in BEAS-2B cells with berberine ( $1 \mu \mathrm{M})$ pre-treatment. (A) Expression levels of $I \kappa B, p-I \kappa B$, and $p 65$ proteins were analyzed using western blotting $(20 \mu g$ per sample). The relative quantity of $(B) I \kappa B(n=6),(C) p-I \kappa B(n=3)$ and $(D)$ nucleus $p 65(n=6)$ was normalized to $\beta$-actin and PCNA, respectively. $\beta$-actin and PCNA expression was used as an internal control. Results are presented as mean \pm SEM. -, cell only; S, pro-inflammation cytokine stimulation; D, DMSO, B, berberine.

were not caused by JNK or ERK activation by adding MAP kinase inhibitors. Thus, whether berberine is able to ameliorate the asthmatic symptoms by reducing IL-6 and CCL11 secretion of airway epithelial cells in vivo is needed to further investigate. In conclusion, berberine perhaps is able to relieve airway inflammation by suppressing cytokine and chemokine production of epithelial cells.

\section{Abbreviations}

Th, T helper; IL, Interleukin; AHR, Airway hyperresponsiveness; EBC, Exhaled breath condensate; TNF, Tumor necrosis factor; MAPK, Mitogen-activated protein kinase; NF- $\mathrm{BB}$, Nuclear factor-kappa B; IкB, Inhibitor of kappa B; JNK, c-Jun NH2-terminal kinase; ERK, Extracellular signalregulated kinase; DMSO, Dimethyl sulfoxide; CCK-8, Cell Counting-kit 8; TSLP, Thymic stromal lymphopoietin; OVA, Ovalbumin.

\section{Supplementary Material}

Supplementary figures and tables.

http://www.medsci.org/v17p1464s1.pdf

\section{Acknowledgments}

This work was supported in part by a grant from Ministry of Science of Technology, Taiwan (R.O.C.): MOST 107-2320-B-182-005-MY3; and grants from Chang Gung Memorial Hospital: CMRPG1A0071 3, CMRPG1E0011 3, CMRPG1G0021, CMRPD1I0081 2, and BMRP 362.

\section{Competing Interests}

The authors have declared that no competing interest exists.

\section{References}

1. To T, Stanojevic S, Moores G, Gershon AS, Bateman ED, Cruz AA, et al. Global asthma prevalence in adults: findings from the cross-sectional world health survey. BMC Public Health. 2012; 12: 204.

2. Holgate ST. Innate and adaptive immune responses in asthma. Nature Medicine. 2012; 18: 673-83. 
3. Lambrecht BN, Hammad H. The airway epithelium in asthma. Nature Medicine. 2012; 18: 684-92.

4. Li J, Zhang F, Li J. The immunoregulatory effects of traditional Chinese medicine on treatment of asthma or asthmatic inflammation. The American Journal of Chinese Medicine. 2015; 43: 1059-81.

5. Lloyd CM, Saglani S. Epithelial cytokines and pulmonary allergic inflammation. Current Opinion in Immunology. 2015; 34: 52-8.

6. Lloyd CM, Hessel EM. Functions of $T$ cells in asthma: more than just $\mathrm{T}(\mathrm{H}) 2$ cells. Nature Reviews Immunology. 2010; 10: 838-48.

7. Smit JJ, Lukacs NW. A closer look at chemokines and their role in asthmatic responses. European Journal of Pharmacology. 2006; 533: 277-88.

8. Shamri R, Melo RC, Young KM, Bivas-Benita M, Xenakis JJ, Spencer LA, et al. CCL11 elicits secretion of RNases from mouse eosinophils and their cell-free granules. FASEB Journal. 2012; 26: 2084-93.

9. Wu D, Zhou J, Bi H, Li L, Gao W, Huang M, et al. CCL11 as a potential diagnostic marker for asthma? The Journal of Asthma. 2014; 51: 847-54

10. Wu CJ, Huang WC, Chen LC, Shen CR, Kuo ML. Pseudotyped adeno-associated virus 2/9-delivered CCL11 shRNA alleviates lung inflammation in an allergen-sensitized mouse model. Human Gene Therapy. 2012; 23: 1156-65.

11. Yokoyama A, Kohno N, Fujino S, Hamada H, Inoue Y, Fujioka S, et al. Circulating interleukin-6 levels in patients with bronchial asthma. American Journal of Respiratory and Critical Care Medicine. 1995; 151: 1354-8.

12. Rincon M, Irvin CG. Role of IL-6 in asthma and other inflammatory pulmonary diseases. International Journal of Biological Sciences. 2012; 8: 1281-90.

13. Scheller J, Chalaris A, Schmidt-Arras D, Rose-John S. The pro- and anti-inflammatory properties of the cytokine interleukin-6. Biochimica et Biophysica Acta. 2011; 1813: 878-88

14. Rincon M, Anguita J, Nakamura T, Fikrig E, Flavell RA. Interleukin (IL)-6 directs the differentiation of IL-4-producing CD4+ $\mathrm{T}$ cells. Journal of Experimental Medicine. 1997; 185: 461-9.

15. Heijink IH, Vellenga E, Borger P, Postma DS, de Monchy JG, Kauffman HF. Interleukin- 6 promotes the production of interleukin- 4 and interleukin- 5 by interleukin-2-dependent and -independent mechanisms in freshly isolated human T cells. Immunology. 2002; 107: 316-24.

16. Neveu WA, Allard JL, Raymond DM, Bourassa LM, Burns SM, Bunn JY, et al. Elevation of IL-6 in the allergic asthmatic airway is independent of inflammation but associates with loss of central airway function. Respiratory Research. 2010; 11: 28.

17. Hoeck J, Woisetschlager M. STAT6 mediates eotaxin-1 expression in IL-4 or TNF-alpha-induced fibroblasts. Journal of Immunology. 2001; 166: 4507-15.

18. Yoshifuku K, Matsune S, Ohori J, Sagara Y, Fukuiwa T, Kurono Y. IL-4 and TNF-alpha increased the secretion of eotaxin from cultured fibroblasts of nasal polyps with eosinophil infiltration. Rhinology. 2007; 45: 235-41.

19. Matsukura S, Stellato C, Plitt JR, Bickel C, Miura K, Georas SN, et al. Activation of eotaxin gene transcription by NF-kappa B and STAT6 in human airway epithelial cells. Journal of Immunology. 1999; 163: 6876-83.

20. Gloire G, Legrand-Poels S, Piette J. NF-kappaB activation by reactive oxygen species: fifteen years later. Biochemical Pharmacology. 2006; 72: 1493-505.

21. Sabio G, Davis RJ. TNF and MAP kinase signalling pathways. Seminars in Immunology. 2014; 26: 237-45.

22. Chang L, Karin M. Mammalian MAP kinase signalling cascades. Nature. 2001; 410: $37-40$.

23. Cicero AF, Baggioni A. Berberine and its role in chronic disease. Advances in Experimental Medicine and Biology. 2016; 928: 27-45.

24. Lee $\mathrm{CH}$, Chen JC, Hsiang CY, Wu SL, Wu HC, Ho TY. Berberine suppresses inflammatory agents-induced interleukin-1beta and tumor necrosis factor-alpha productions via the inhibition of IkappaB degradation in human lung cells. Pharmacological Research. 2007; 56: 193-201.

25. Yan F, Wang L, Shi Y, Cao H, Liu L, Washington MK, et al. Berberine promotes recovery of colitis and inhibits inflammatory responses in colonic macrophages and epithelial cells in DSS-treated mice. American Journal of Physiology Gastrointestinal and Liver Physiology. 2012; 302: G504-14.

26. Wang $\mathrm{Q}$, Qi J, Hu R, Chen Y, Kijlstra A, Yang P. Effect of berberine on proinflammatory cytokine production by ARPE-19 cells following stimulation with tumor necrosis factor-alpha. Investigative Ophthalmology \& Visual Science. 2012; 53: 2395-402

27. Li XM. Traditional Chinese herbal remedies for asthma and food allergy. The Journal of Allergy and Clinical Immunology. 2007; 120: 25-31.

28. Chan CC, Liou CJ, Xu PY, Shen JJ, Kuo ML, Len WB, et al. Effect of dehydroepiandrosterone on atopic dermatitis-like skin lesions induced by 1-chloro-2,4-dinitrobenzene in mouse. Journal of Dermatological Science. 2013; 72: $149-57$

29. Chan CK, Kuo ML, Shen JJ, See LC, Chang HH, Huang JL. Ding Chuan Tang, a Chinese herb decoction, could improve airway hyper-responsiveness in stabilized asthmatic children: a randomized, double-blind clinical trial. Pediatric Allergy and Immunology. 2006; 17: 316-22.

30. Castro-Rodriguez JA, Rodrigo GJ. A systematic review of long-acting beta2-agonists versus higher doses of inhaled corticosteroids in asthma. Pediatrics. 2012; 130: e650-7.

31. Luhadia SK. Steroid resistant asthma. The Journal of The Association of Physicians of India. 2014; 62: 38-40.

32. Mark JD. Integrative medicine and asthma. Pediatric Clinics of North America. 2007; 54: 1007-23; xii.
33. Kao ST, Yeh TI, Hsieh CC, Shiau HB, Yeh FT, Lin JG. The effects of Ma-Xing-Gan-Shi-Tang on respiratory resistance and airway leukocyte infiltration in asthmatic guinea pigs. Immunopharmacology and Immunotoxicology. 2001; 23: 445-58.

34. Wang SD, Lin LJ, Chen CL, Lee SC, Lin CC, Wang JY, et al. Xiao-Qing-Long-Tang attenuates allergic airway inflammation and remodeling in repetitive Dermatogoides pteronyssinus challenged chronic asthmatic mice model. Journal of Ethnopharmacology. 2012; 142: 531-8.

35. Fleming RM. Safety of ephedra and related anorexic medications. Expert Opinion on Drug Safety. 2008; 7: 749-59.

36. Huang WC, Fang LW, Liou CJ. Phloretin attenuates allergic airway inflammation and oxidative stress in asthmatic mice. Frontiers in Immunology. 2017; 8: 134.

37. Busse PJ, Schofield B, Birmingham N, Yang N, Wen MC, Zhang T, et al. The traditional Chinese herbal formula ASHMI inhibits allergic lung inflammation in antigen-sensitized and antigen-challenged aged mice. Annals of Allergy, Asthma \& Immunology. 2010; 104: 236-46.

38. Li XM, Huang CK, Zhang TF, Teper AA, Srivastava K, Schofield BH, et al. The chinese herbal medicine formula MSSM-002 suppresses allergic airway hyperreactivity and modulates $\mathrm{TH} 1 / \mathrm{TH} 2$ responses in a murine model of allergic asthma. The Journal of Allergy and Clinical Immunology. 2000; 106: 660-8

39. Ebeling C, Wu Y, Skappak C, Gordon JR, Ilarraza R, Adamko DJ. Compound CVT-E002 attenuates allergen-induced airway inflammation and airway hyperresponsiveness, in vivo. Molecular Nutrition \& Food Research. 2011; 55: 1905-8.

40. Kelly-Pieper K, Patil SP, Busse P, Yang N, Sampson H, Li XM, et al. Safety and tolerability of an antiasthma herbal formula (ASHMI) in adult subjects with asthma: a randomized, double-blinded, placebo-controlled, dose-escalation phase I study. Journal of Alternative and Complementary Medicine. 2009; 15: 735-43.

41. Watson RR, Zibadi S, Rafatpanah H, Jabbari F, Ghasemi R, Ghafari J, et al. Oral administration of the purple passion fruit peel extract reduces wheeze and cough and improves shortness of breath in adults with asthma. Nutrition Research. 2008; 28: 166-71.

42. Boskabady MH, Javan $H$, Sajady M, Rakhshandeh $H$. The possible prophylactic effect of Nigella sativa seed extract in asthmatic patients. Fundamental and Clinical Pharmacology 2007; 21: 559-66.

43. Xu L, Li J, Zhang Y, Zhao P, Zhang X. Regulatory effect of baicalin on the imbalance of Th17/Treg responses in mice with allergic asthma. Journal of Ethnopharmacology. 2017; 208: 199-206.

44. Vafa Amirkia MH. Alkaloids as drug leads - A predictive structural and biodiversity-based analysis. Phytochemistry Letters. 2014; 10: xlviii-liii.

45. Tillhon M, Guaman Ortiz LM, Lombardi P, Scovassi AI. Berberine: new perspectives for old remedies. Biochemical Pharmacology. 2012; 84: 1260-7.

46. Shen $\mathrm{CH}$, Stavnezer J. Interaction of stat6 and NF-kappaB: direct association and synergistic activation of interleukin-4-induced transcription. Molecular and Cellular Biology. 1998; 18: 3395-404.

47. Kim BH, Kim M, Yin $\mathrm{CH}$, Jee JG, Sandoval $\mathrm{C}$, Lee $\mathrm{H}$, et al. Inhibition of the signalling kinase JAK3 alleviates inflammation in monoarthritic rats. The British Journal of Pharmacology. 2011; 164: 106-18.

48. Li Z, Zheng J, Zhang N, Li C. Berberine improves airway inflammation and inhibits NF-kappaB signaling pathway in an ovalbumin-induced rat model of asthma. The Journal of Asthma. 2016; 53: 999-1005.

49. Moon PD, Choi $\mathrm{IH}$, Kim HM. Berberine inhibits the production of thymic stromal lymphopoietin by the blockade of caspase-1/NF-kappaB pathway in mast cells. International Immunopharmacology. 2011; 11: 1954-9.

50. Kang BY, Chung SW, Cho D, Kim TS. Involvement of p38 mitogen-activated protein kinase in the induction of interleukin-12 p40 production in mouse macrophages by berberine, a benzodioxoloquinolizine alkaloid. Biochemical Pharmacology. 2002; 63: 1901-10. 